\title{
Approximation by Max-Product Sampling Operators Based on Sinc-Type Kernels
}

\author{
Lucian Coroianu and Sorin G. Gal \\ Department of Mathematics and Computer Science, \\ The University of Oradea \\ Universitatii 1, 410087 \\ Oradea, Romania \\ lcoroianu@uoradea.ro and galso@uoradea.ro
}

\begin{abstract}
By using the so-called max-product method, in this paper we associate to the truncated Whittaker linear operator and to the non-truncated and truncated linear sampling operators based on the Fejér-type kernel, nonlinear sampling operators for which Jackson-type approximation orders in terms of the first order modulus of continuity are obtained, thus essentially improving the orders of approximation given by their linear correspondents. Also, we prove that the max-product truncated Whittaker operator and the max-product truncated operator based on the Fejér-type kernel, partially preserve the monotonicity.
\end{abstract}

Key words and phrases : Sampling theory, signal theory, nonlinear truncated Whittaker operator, Fejér-type kernel, nonlinear sampling operators of max-product kind, Jackson-type estimates, shape preserving property.

2000 AMS Mathematics Subject Classification - Primary 94A20, 94A12, 41A35; Secondary 41A25, 41A20.

\section{Introduction}

The sinc-approximation operators were first introduced and studied in [15], [6] and [20] under the name of cardinal function and of truncated cardinal function. Later on, these linear approximation operators were intensively studied in e.g. [1]-[3], [7]-[10], [13], [14], [16], [17], [18], [19] (see also the references cited there).

Based on the Open Problem 5.5.4, pp. 324-326 in [12], in a series of recent papers submitted for publication we have introduced and studied the so-called max-product operators attached to the Bernstein polynomials and to other linear Bernstein-type operators, like those of Favard-Szász-Mirakjan operators (truncated and nontruncated case), Baskakov operators (truncated and nontruncated case), Meyer-König and Zeller operators and Bleimann-Butzer-Hahn operators. 
This idea applied, for example, to the linear Bernstein operators $B_{n}(f)(x)=$ $\sum_{k=0}^{n} p_{n, k}(x) f(k / n)$, where $p_{n, k}(x)=\left(\begin{array}{l}n \\ k\end{array}\right) x^{k}(1-x)^{n-k}$, works as follows. Writing in the equivalent form $B_{n}(f)(x)=\frac{\sum_{k=0}^{n} p_{n, k}(x) f(k / n)}{\sum_{k=0}^{n} p_{n, k}(x)}$ and then replacing the sum operator $\Sigma$ by the maximum operator $\bigvee$, one obtains the nonlinear Bernstein operator of max-product kind

$$
B_{n}^{(M)}(f)(x)=\frac{\bigvee_{k=0}^{n} p_{n, k}(x) f\left(\frac{k}{n}\right)}{\bigvee_{k=0}^{n} p_{n, k}(x)},
$$

where the notation $\bigvee_{k=0}^{n} p_{n, k}(x)$ means $\max \left\{p_{n, k}(x) ; k \in\{0, \ldots, n\}\right\}$ and similarly for the numerator.

For this max-product operator nice approximation and shape preserving properties were found in e.g. [5].

In the very recent paper [11], applying this idea to the Whittaker's cardinal series, we obtained a Jackson-type estimate in approximation of $f$ by the maxproduct Whittaker sampling operator given by

$$
S_{W, \varphi}^{(M)}(f)(t)=\frac{\bigvee_{k=-\infty}^{\infty} \varphi(W t-k) f\left(\frac{k}{W}\right)}{\bigvee_{k=-\infty}^{\infty} \varphi(W t-k)}, t \in \mathbb{R},
$$

where $W>0$ and $\varphi$ is a kernel given by the formula $\varphi(t)=\operatorname{sinc}(t)=\frac{\sin (\pi t)}{\pi t}$.

The first aim of the present paper is to apply a similar idea to the sampling operator based on the Fejér-type kernel and to obtain a Jackson-type estimate in approximation of $f$ by the max-product operator given by $(1)$ with $\varphi(t)=$ $\frac{1}{2} \cdot[\operatorname{sinc}(t / 2)]^{2}$.

The second aim is to apply the max-product idea to the truncated Whittaker series defined by (see e.g. Borel [6], Whittaker [20])

$$
W_{n}(f)(x)=\sum_{k=0}^{n} \frac{\sin (n x-k \pi)}{n x-k \pi} \cdot f\left(\frac{k \pi}{n}\right), x \in[0, \pi],
$$

and to the truncated sampling operator based on the Fejér's kernel and defined by

$$
T_{n}(f)(x)=\sum_{k=0}^{n} \frac{\sin ^{2}(n x-k \pi)}{(n x-k \pi)^{2}} \cdot f\left(\frac{k \pi}{n}\right), x \in[0, \pi]
$$


thus introducing and studying the approximation properties of the truncated max-product Whittaker operator given by

$$
W_{n}^{(M)}(f)(x)=\frac{\bigvee_{k=0}^{n} \frac{\sin (n x-k \pi)}{n x-k \pi} \cdot f\left(\frac{k \pi}{n}\right)}{\bigvee_{k=0}^{n} \frac{\sin (n x-k \pi)}{n x-k \pi}}, x \in[0, \pi]
$$

and of the truncated max-product operator based on the Fejér kernel, given by

$$
T_{n}^{(M)}(f)(x)=\frac{\bigvee_{k=0}^{n} \frac{\sin ^{2}(n x-k \pi)}{(n x-k \pi)^{2}} \cdot f\left(\frac{k \pi}{n}\right)}{\bigvee_{k=0}^{n} \frac{\sin ^{2}(n x-k \pi)}{(n x-k \pi)^{2}}}, x \in[0, \pi]
$$

Here by convention we take $\sin (0) / 0=1$, which means that for every $x=k \pi / n$, $k \in\{0,1, \ldots, n\}$, we take $\frac{\sin (n x-k \pi)}{n x-k \pi}=1$.

The plan of the paper goes as follows : in Section 2 we deal with the maxproduct sampling operator based on the Fejér kernel, Section 3 studies the truncated max-product Whittaker operator, while in Section 4 we deal with the truncated max-product operator based on the Fejér kernel.

In every section we compare the approximation results for the max-product operators with those for the corresponding linear operators.

\section{Max-Product Operator Based on Fejér Kernel}

In this section we deal with the approximation properties of the max-product sampling operator $S_{W, \varphi}^{(M)}(f)$ based on the Fejér kernel, given by the formula (1) in Introduction with $\varphi(x)=\frac{1}{2} \cdot \operatorname{sinc}^{2}(x / 2)$.

Let us denote

$$
C B_{+}(\mathbb{R})=\left\{f: \mathbb{R} \rightarrow \mathbb{R}_{+} ; f \text { is bounded and continuous on } \mathbb{R}\right\} .
$$

Remark. If we take in $(1) \varphi(t)=\frac{1}{2} \cdot \sin c^{2}(t / 2)$ for all $t \in \mathbb{R}$ and $f \in$ $C B_{+}(\mathbb{R})$, then $S_{W, \varphi}^{(M)}(f)(t)$ is a well-defined function for all $t \in \mathbb{R}$. Indeed, denoting $s_{W, k}(t)=\varphi(W t-k)=\frac{1}{2} \cdot \frac{\sin ^{2} \pi(W t-k) / 2}{\pi^{2}(W t-k)^{2} / 4}$, it is immediate that for $t \in(j / W,(j+1) / W)$ we have $\pi(W t-j) / 2 \in(0, \pi / 2)$ and consequently we get $s_{W, j}(t)>0$ for all $t \in(j / W,(j+1) / W)$. This implies $\bigvee_{k=-\infty}^{\infty} \varphi(W t-k)=$ $\bigvee_{k=-\infty}^{\infty} s_{W, k}(t) \geq s_{W, j}(t)>0$ for all $t \in(j / W,(j+1) / W)$. Now, if $t=j / W$ then since $\operatorname{sinc}(0)=1$ we obtain $s_{W, j}(j / W)=1 / 2$, which immediately implies $\bigvee_{k=-\infty}^{\infty} s_{W, k}(j / W) \geq 1 / 2$. Also, for $t=(j+1) / W$ we get $s_{W, j}((j+1) / W)=\frac{2}{\pi^{2}}$, which immediately implies $\bigvee_{k=-\infty}^{\infty} s_{W, k}(j / W) \geq 2 / \pi^{2}$. From the above reasonings it follows that $\bigvee_{k=-\infty}^{\infty} \varphi(W t-k)>0$ for all $t \in[j / W,(j+1) / W]$ and $j \in \mathbb{Z}$. Clearly, this implies $\bigvee_{k=-\infty}^{\infty} \varphi(W t-k)>0$ for all $t \in \mathbb{R}$. 

$t \in \mathbb{R})$

Also, since $f \in C B_{+}(\mathbb{R})$, by taking $C \geq f(t)$ for all $t \in \mathbb{R}$, we get (for all

$$
\begin{aligned}
0 \leq & S_{W, \varphi}^{(M)}(f)(t) \\
& \bigvee_{k=-\infty}^{\infty} \varphi(W t-k) f\left(\frac{k}{W}\right) \\
\bigvee_{k=-\infty}^{\infty} \varphi(W t-k) & \bigvee_{k=-\infty}^{\infty} \varphi(W t-k) \bigvee_{k=-\infty}^{\infty} f\left(\frac{k}{W}\right) \\
& =\bigvee_{k=-\infty}^{\infty} f\left(\frac{k}{W}\right) \leq C,
\end{aligned}
$$

therefore $S_{W, \varphi}^{(M)}(f)(t) \in \mathbb{R}$ for all $t \in \mathbb{R}$. In addition, it is immediate that $S_{W, \varphi}^{(M)}\left(e_{0}\right)=e_{0}$ where $e_{0}(t)=1$ for all $t \in \mathbb{R}$.

Lemma 2.1. Let $S_{W, \varphi}^{(M)}$ be as above, with $\varphi(t)=\frac{1}{2} \cdot \operatorname{sinc}^{2}(t / 2)$ for all $t \in \mathbb{R}$.

(i) We have $S_{W, \varphi}^{(M)}: C B_{+}(\mathbb{R}) \rightarrow C B_{+}(\mathbb{R})$, for all $W>0$;

(ii) If $f, g \in C B_{+}(\mathbb{R})$ satisfy $f \leq g$ then $S_{W, \varphi}^{(M)}(f) \leq S_{W, \varphi}^{(M)}(g)$ for all $W>0$;

(iii) $S_{W, \varphi}^{(M)}(f+g) \leq S_{W, \varphi}^{(M)}(f)+S_{W, \varphi}^{(M)}(g)$ for all $f, g \in C B_{+}(\mathbb{R})$ and $W>0$;

(iv) For all $f, g \in C B_{+}(\mathbb{R}), W>0$ and $t \in \mathbb{R}$ we have

$$
\left|S_{W, \varphi}^{(M)}(f)(t)-S_{W, \varphi}^{(M)}(g)(t)\right| \leq S_{W, \varphi}^{(M)}(|f-g|)(t)
$$

(v) $S_{W, \varphi}^{(M)}$ is positively homogenous, that is $S_{W, \varphi}^{(M)}(\lambda f)=\lambda S_{W, \varphi}^{(M)}(f)$ for all $\lambda \geq 0, W>0$ and $f \in C B_{+}(\mathbb{R})$.

Proof. The proof follows word for word the lines in the proof of Lemma 2.3 in [11], with two differences : in the formula $s_{W, k}(t)=\varphi(W t-k)$, instead of $\varphi(t)=\sin c(t)$ we choose $\varphi(t)=\frac{1}{2} \operatorname{sinc}^{2}(t / 2)$ and the value $\bigvee_{|k|>n} \frac{1}{\pi(W t-k)}$ in [11], appears to be here $\bigvee_{|k|>n} \frac{1}{(W t-k)^{2}}$.

We use this occasion to mention here some small inadvertence in the proof of Lemma 2.3 in [11]. Thus, in the first part of the proof of Lemma 2.3, (i) in [11], in fact it is proved the continuity of denominator (and not of the numerator, as it is stated wrongly there). Consequently, clearly that on the page 64 of [11], line 6 from below, the value $f(k / W)$ must be deleted from that formula and $M$ must be replaced there by 1 , while in the last phrase of the proof of the point (i), the word "denominator" must be interchanged with the word "numerator".

From the definition of $S_{W, \varphi}^{(M)}$ it is easy to see that instead of (ii), $S_{W, \varphi}^{(M)}$ satisfies the stronger condition

$$
S_{W, \varphi}^{(M)}(f \vee g)(x)=S_{W, \varphi}^{(M)}(f)(x) \vee S_{W, \varphi}^{(M)}(g)(x), f, g \in C B_{+}(\mathbb{R}) .
$$


Corollary 2.2. Let $\varphi(t)=\frac{1}{2} \cdot \operatorname{sinc}^{2}(t / 2)$ for all $t \in \mathbb{R}$. For all $f \in C B_{+}(\mathbb{R})$, $W>0$ and $t \in \mathbb{R}$ we have

$$
\left|f(t)-S_{W, \varphi}^{(M)}(f)(t)\right| \leq\left[\frac{1}{\delta} S_{W, \varphi}^{(M)}\left(\Phi_{t}\right)(t)+1\right] \omega_{1}(f ; \delta)_{\mathbb{R}},
$$

where $\delta>0, \Phi_{t}(u)=|t-u|$ for all $t, u \in \mathbb{R}$, and $\omega_{1}(f ; \delta)_{\mathbb{R}}=\max \{\mid f(x)-$ $f(y)|; x, y \in \mathbb{R}| x-y \mid, \leq \delta\}$.

Proof. The proof is similar with the lines in the proof of Corollary $2.2 \mathrm{in}$ [11].

In order to obtain approximation results of the operator $S_{W, \varphi}^{(M)}$, first we need to find a simpler form for the expression $\bigvee_{k=-\infty}^{\infty} \varphi(W t-k)$.

Lemma 2.3. Let $\varphi(t)=\frac{1}{2} \cdot \operatorname{sinc}^{2}(t / 2)$, for all $t \in \mathbb{R}$. Then for all $j \in \mathbb{Z}$ we have

$$
\bigvee_{k=-\infty}^{\infty} \varphi(W t-k)=\max \{\varphi(W t-j), \varphi(W t-j-1)\}, t \in\left[\frac{j}{W}, \frac{j+1}{W}\right]
$$

Proof. Let us fix $t \in[j / W,(j+1) / W]$. It is easy to check that $\pi(W t-j) / 2 \in$ $[0, \pi / 2]$ and by the well-known inequality $\sin x \geq \frac{2 x}{\pi}$ for all $x \in[0, \pi / 2]$ we get $\frac{\sin ^{2} \pi(W t-j) / 2}{\pi^{2}(W t-j)^{2} / 4} \geq \frac{4}{\pi^{2}}$ which implies $\varphi(W t-j) \geq \frac{2}{\pi^{2}}$. On the other hand, it is immediate that for $k \in \mathbb{Z} \backslash\{j, j+1\}$ we have $(W t-k)^{2} \geq 1$ which implies

$$
\frac{\sin ^{2} \pi(W t-k) / 2}{\pi^{2}(W t-k)^{2} / 4} \leq \frac{1}{\pi^{2}(W t-k)^{2} / 4} \leq \frac{4}{\pi^{2}} .
$$

We thus obtain $\varphi(W t-k) \leq \frac{2}{\pi^{2}}$. Clearly, this implies

$$
\bigvee_{k=-\infty}^{\infty} \varphi(W t-k)=\max \{\varphi(W t-j), \varphi(W t-j-1)\}
$$

which proves the lemma

In what follows, we study the approximation properties of the max-product operator $S_{W, \varphi}^{(M)}(f)$. From Corollary 2.2. it follows that it is enough to get a good estimate for the expression

$$
E_{W, \varphi}(t)=S_{W, \varphi}^{(M)}\left(\Phi_{t}\right)(t)=\frac{\bigvee_{k=-\infty}^{\infty} \varphi(W t-k)\left|\frac{k}{W}-t\right|}{\bigvee_{k=-\infty}^{\infty} \varphi(W t-k)} .
$$

Theorem 2.4. Let $\varphi(t)=\frac{1}{2} \cdot \operatorname{sinc}^{2}(t / 2)$, for all $t \in \mathbb{R}$ and $f \in C B_{+}(\mathbb{R})$. Then

$$
\left|S_{W, \varphi}^{(M)}(f)(t)-f(t)\right| \leq 4 \omega_{1}\left(f ; \frac{1}{W}\right)_{\mathbb{R}}, \text { for all } t \in \mathbb{R} .
$$


Here recall that $\omega_{1}(f ; \delta)_{\mathbb{R}}=\sup \{|f(u)-f(v)| ; u, v \in \mathbb{R},|u-v| \leq \delta\}$.

Proof. Let $t \in \mathbb{R}$ and let $j \in \mathbb{Z}$ such that $t \in\left[\frac{j}{W}, \frac{j+1}{W}\right]$. By Lemma 2.3. we have

$$
\begin{aligned}
& E_{W, \varphi}(t)=\frac{\bigvee_{k=-\infty}^{\infty} \varphi(W t-k)\left|\frac{k}{W}-t\right|}{\bigvee_{k=-\infty}^{\infty} \varphi(W t-k)} \leq \frac{\bigvee_{k=-\infty}^{\infty} \varphi(W t-k)|t-k / W|}{\varphi(W t-j)} \\
& =\frac{\bigvee_{k=-\infty}^{\infty} \frac{1}{2} \cdot \frac{\sin ^{2} \pi(W t-k) / 2}{\pi^{2}(W t-k)^{2} / 4}|t-k / W|}{\frac{1}{2} \cdot \frac{\sin ^{2} \pi(W t-j) / 2}{\pi^{2}(W t-j)^{2} / 4}} \\
& \frac{\bigvee_{k=-\infty}^{\infty} \frac{\sin ^{2} \pi(W t-k) / 2}{\pi^{2}(W t-k)^{2} / 4}|t-k / W|}{\frac{\sin ^{2} \pi(W t-j) / 2}{\pi^{2}(W t-j)^{2} / 4}} \leq \frac{\bigvee_{k=-\infty}^{\infty} \frac{|\sin \pi(W t-k) / 2|}{\pi|W t-k| / 2}|t-k / W|}{\frac{\sin ^{2} \pi(W t-j) / 2}{\pi^{2}(W t-j)^{2} / 4}} \\
& \leq \frac{\bigvee_{k=-\infty}^{\infty} \frac{1}{\pi|W t-k| / 2}|t-k / W|}{\frac{\sin ^{2} \pi(W t-j) / 2}{\pi^{2}(W t-j)^{2} / 4}}=\frac{\pi}{2 W} \cdot \frac{(W t-j)^{2}}{\sin ^{2} \pi(W t-j) / 2} \\
& \leq \frac{\pi}{2 W} \cdot \frac{(W t-j)^{2}}{(\pi(W t-j) / 2)^{2} \cdot\left(4 / \pi^{2}\right)}=\frac{\pi}{2 W}
\end{aligned}
$$

where in the last inequality we used again the formula $\sin x \geq \frac{2 x}{\pi}$ for all $x \in\left[0, \frac{\pi}{2}\right]$ since for $t \in\left[\frac{j}{W}, \frac{j+1}{W}\right]$ it follows that $\pi(W t-j) / 2 \in\left[0, \frac{\pi}{2}\right]$.

From the above inequality, applying Corollary 2.2 for $\delta=\frac{\pi}{2 W}$ and from the property $\omega_{1}(f, \lambda \delta)_{\mathbb{R}} \leq([\lambda]+1) \omega_{1}(f, \delta)_{\mathbb{R}}$ we obtain the desired conclusion.

Remarks. 1) In the case of Theorem 2.4, the order of approximation of the expression $E_{W, \varphi}(t)$ cannot be improved. For this purpose let us take $t=$ $\frac{1}{2 W}$. Lemma 2.3 implies $\bigvee_{k=-\infty}^{\infty} \varphi(W t-k)=\max \{\varphi(W t), \varphi(W t-1)\}$. But it is immediate that for $t=1 / 2 W$ we have $\varphi(W t)=\varphi(W t-1)=\frac{4}{\pi^{2}}$. Since 


$$
\begin{aligned}
& \bigvee_{k=-\infty}^{\infty} \varphi(W t-k)\left|\frac{k}{W}-t\right| \geq \varphi(W t)|t|, \text { for } t=1 /(2 W) \text { we get } \\
& E_{W, \varphi}(t)=\frac{\bigvee_{k=-\infty}^{\infty} \varphi(W t-k)\left|\frac{k}{W}-t\right|}{\bigvee_{k=-\infty}^{\infty} \varphi(W t-k)}=\frac{\bigvee_{k=-\infty}^{\infty} \varphi(W t-k)\left|\frac{k}{W}-t\right|}{\varphi(W t)} \\
& \geq \frac{\varphi(W t)|t|}{\varphi(W t)}=\frac{1}{2 W} .
\end{aligned}
$$

This implies the desired conclusion.

2) Regarding the estimate in approximation by the linear sampling operator based on the Fejér-type kernel introduced in [18], it is known that we do not have even an estimate in terms of the first modulus of continuity (see e.g. the Example after Theorem 4 in [14]). Therefore, according to the above Theorem 2.4 , the error estimate given by the max-product operator based on the Fejértype kernel is essentially better than that given by its linear correspondent.

\section{Truncated Max-Product Whittaker Operator}

In this section we deal with the approximation and shape preserving properties of the truncated Whittaker operator of max-product kind, $W_{n}^{(M)}(f)$ given by the formula (2) in Introduction.

Remark. Note that since by convention we take $\sin (0) / 0=1$, denoting $s_{n, k}(x)=\frac{\sin (n x-k \pi)}{n x-k \pi}$, it is clear that by the obvious property $s_{n, k}(j \pi / n)=1$ if $k=j$ and $s_{n, k}(j \pi / n)=0$ if $k \neq j$, we immediately obtain the interpolation properties $W_{n}^{(M)}(f)(j \pi / n)=f(j \pi / n)$, for all $j \in\{0, \ldots, n\}$.

Also, it is clear that $W_{n}^{(M)}(f)$ is a well-defined function for all $x \in[0, \pi]$ and it is continuous on $[0, \pi]$. Indeed, if $x=j \pi / n$ then by $s_{n, j}(j \pi / n)=1$ it follows that $\bigvee_{k=0}^{n} s_{n, k}(j \pi / n) \geq 1>0$, for all $j \in\{0,1, \ldots, n\}$. Now, if $x \in(j \pi / n,(j+1) \pi / n)$ with $j \in\{0,1, \ldots, n\}$ arbitrary fixed, we easily obtain that $n x-j \pi \in(0, \pi)$ and therefore $s_{n, j}(x)=\frac{\sin (n x-j \pi)}{n x-j \pi}>0$. In conclusion, $W_{n}^{(M)}(f)$ is a well-defined function for all $x \in[0, \pi]$ and because both the denominator and numerator are continuous functions as maximum of a finite number of continuous functions, it follows the continuity of $W_{n}^{(M)}(f)$ on the interval $[0, \pi]$.

In addition, note that we evidently have $W_{n}\left(e_{0}\right)(x)=e_{0}(x)$, where $e_{0}(x)=1$, for all $x \in[0, \pi]$.

Let us define the space

$$
C_{+}[0, \pi]=\left\{f:[0, \pi] \rightarrow \mathbb{R}_{+} ; f \text { is continuous on }[0, \pi]\right\} .
$$


In what follows, we will see that for $f \in C_{+}[0, \pi]$, the $W_{n}^{(M)}$ operator fulfils similar properties with those of the $B_{n}^{(M)}$ operator in [4].

Lemma 3.1. (i) We have $W_{n}^{(M)}: C_{+}[0, \pi] \rightarrow C_{+}[0, \pi]$, for all $n \in \mathbb{N}$;

(ii) If $f, g \in C_{+}[0, \pi]$ satisfy $f \leq g$ then $W_{n}^{(M)}(f) \leq W_{n}^{(M)}(g)$ for all $n \in N$ ;

(iii) $W_{n}^{(M)}(f+g) \leq W_{n}^{(M)}(f)+W_{n}^{(M)}(g)$ for all $f, g \in C_{+}[0, \pi]$;

(iv) For all $f, g \in C_{+}[0, \pi], n \in N$ and $x \in[0, \pi]$ we have

$$
\left|W_{n}^{(M)}(f)(x)-W_{n}^{(M)}(g)(x)\right| \leq W_{n}^{(M)}(|f-g|)(x) ;
$$

(v) $W_{n}^{(M)}$ is positively homogenous, that is $W_{n}^{(M)}(\lambda f)=\lambda W_{n}^{(M)}(f)$ for all $\lambda \geq 0$ and $f \in C_{+}[0, \pi]$.

Proof. (i) The continuity of $W_{n}^{(M)}(f)$ on $[0, \pi]$ follows from the previous Remark. It remains to prove the positivity of $W_{n}^{(M)}(f)$. So let $f:[0, \pi] \rightarrow \mathbb{R}_{+}$ and fix $x \in[0, \pi]$. Reasoning exactly as in the above Remark, there exists $k \in\{0,1, \ldots, n\}$ such that $s_{n, k}(x)>0$. Therefore, denoting $I_{n}^{+}(x)=\{k \in$ $\left.\{0,1, \ldots, n\} ; s_{n, k}(x)>0\right\}$, clearly $I_{n}^{+}(x)$ is nonempty and for $f \in C_{+}[0, \pi]$ we get that

$$
W_{n}^{(M)}(f)(x)=\frac{\bigvee_{k \in I_{n}^{+}(x)} s_{n, k}(x) f\left(x_{n, k}\right)}{\bigvee_{k \in I_{n}^{+}(x)} s_{n, k}(x)} \geq 0 .
$$

The proofs of (ii)-(v) are similar to those for Lemma 2.3 in [11], by taking $W=\frac{\pi}{n}$ and with $\mathbb{R}$ replaced by $[0, \pi]$.

Remark. By (4) it is easy to see that instead of (ii), $W_{n}^{(M)}$ satisfies the stronger condition

$$
W_{n}^{(M)}(f \vee g)(x)=W_{n}^{(M)}(f)(x) \vee W_{n}^{(M)}(g)(x), f, g \in C_{+}[0, \pi] .
$$

Corollary 3.2. For all $f \in C_{+}[0, \pi], n \in N$ and $x \in[0, \pi]$ we have

$$
\left|f(x)-W_{n}^{(M)}(f)(x)\right| \leq\left[\frac{1}{\delta} W_{n}^{(M)}\left(\varphi_{x}\right)(x)+1\right] \omega_{1}(f ; \delta)_{[0, \pi]},
$$

where $\delta>0, \varphi_{x}(t)=|t-x|$ for all $t \in[0, \pi], x \in[0, \pi]$ and $\omega_{1}(f ; \delta)_{[0, \pi]}=$ $\max \{|f(x)-f(y)| ; x, y \in[0, \pi],|x-y| \leq \delta\}$.

Proof. By the above Lemma 3.1 and by the lines in the proof of Corollary 2.2 in [11] (by replacing there $\mathbb{R}$ by $[0, \pi]$ and $W$ by $\frac{\pi}{n}$ ), the proof is immediate.

We can explicitly write the denominator of $W_{n}^{(M)}(f)(x)$, as follows.

Lemma 3.3. Denoting $s_{n, k}(x)=\frac{\sin (n x-k \pi)}{n x-k \pi}$, for any $j \in\{0,1, \ldots, n-1\}$ we have

$$
\bigvee_{k=0}^{n} s_{n, k}(x)=s_{n, j}(x), \text { for all } x \in\left[\frac{j \pi}{n}, \frac{(j+1 / 2) \pi}{n}\right] \text {, }
$$


and

$$
\bigvee_{k=0}^{n} s_{n, k}(x)=s_{n, j+1}(x), \text { for all } x \in\left[\frac{(j+1 / 2) \pi}{n}, \frac{(j+1) \pi}{n}\right] .
$$

Proof. The proof follows word for word the proof of Lemma 3.1, (iv) in [11], by choosing there $W=\frac{\pi}{n}$ and replacing the infinite set $\mathbb{Z}$ by $\{0,1, \ldots, n\}$.

In what follows we study the approximation properties of the max-product operator $W_{n}^{(M)}$. For this purpose, it is enough to get a good estimate for the expression

$$
F_{n}(x):=W_{n}^{(M)}\left(\varphi_{x}\right)(x)=\frac{\bigvee_{k=0}^{n} s_{n, k}(x)\left|x_{n, k}-x\right|}{\bigvee_{k=0}^{n} s_{n, k}(x)}=\frac{\bigvee_{k \in J_{n}^{+}(x)} s_{n, k}(x)\left|x_{n, k}-x\right|}{\bigvee_{k \in J_{n}^{+}(x)} s_{n, k}(x)},
$$

where $I_{n}^{+}(x)=\left\{k \in\{0, \ldots, n\} ; s_{n, k}(x)>0\right\}$ and $x_{n, k}=k \pi / n$, for all $k \in$ $\{0,1, \ldots, n\}$.

In this sense, we present the following.

Theorem 3.4. If $f:[0, \pi] \rightarrow \mathbb{R}_{+}$is continuous then we have the estimate

$$
\left|W_{n}^{(M)}(f)(x)-f(x)\right| \leq 4 \omega_{1}\left(f ; \frac{1}{n}\right)_{[0, \pi]} \text {, for all } n \in \mathbb{N}, n \geq 1, x \in[0, \pi] .
$$

Proof. Firstly, because $W_{n}^{(M)}(f)\left(x_{n, j}\right)=f\left(x_{n, j}\right)$, for all $j \in\{0,1, \ldots, n\}$, in all calculations and estimations we may suppose that $x \neq x_{n, j}$, for all $j \in$ $\{0,1, \ldots, n\}$. Here $x_{n, j}=j \pi / n$, for all $j \in\{0,1, \ldots, n\}$.

Let $x \in[0, \pi]$ and let $j \in[0,1, \ldots, n-1]$ be such that $x \in[j \pi / n,(j+1) \pi / n]$. In order to prove the estimate in the statement we distinguish the following cases: 1) $x \in(j \pi / n,(j+1 / 2) \pi / n]$ and 2$) x \in[(j+1 / 2) \pi / n,(j+1) \pi / n)$.

Case 1) By Lemma 3.3 we get $\bigvee_{k=0}^{n} s_{n, k}(x)=s_{n, j}(x)$ and it follows that

$$
F_{n}(x)=\frac{\bigvee_{k=0}^{n} s_{n, k}(x)\left|x_{n, k}-x\right|}{s_{n, j}(x)}=\frac{\bigvee_{k \in I_{n}^{+}(x)} s_{n, k}(x)\left|x_{n, k}-x\right|}{s_{n, j}(x)} .
$$

Because $j \in I_{n}^{+}(x)$, for all $k \in I_{n}^{+}(x)$ we get

$$
s_{n, k}(x)\left|x_{n, k}-x\right|=\frac{1}{n} \cdot|\sin (n x-k \pi)|=\frac{1}{n} \cdot \sin (n x-j \pi)
$$

and it immediately follows that

$$
F_{n}(x)=\frac{\frac{1}{n} \cdot \sin (n x-j \pi)}{s_{n, j}(x)}=\frac{n x-j \pi}{n} \leq \frac{(n(j+1 / 2) \pi) / n-j \pi}{n}=\frac{\pi}{2 n} .
$$


Case 2) By Lemma 3.3 we get $\bigvee_{k=0}^{n} s_{n, k}(x)=s_{n, j+1}(x)$ and it follows that

$$
F_{n}(x)=\frac{\bigvee_{k=0}^{n} s_{n, k}(x)\left|x_{n, k}-x\right|}{s_{n, j+1}(x)}=\frac{\bigvee_{k \in I_{n}^{+}(x)} s_{n, k}(x)\left|x_{n, k}-x\right|}{s_{n, j+1}(x)}
$$

Since $j+1 \in I_{n}^{+}(x)$, it follows that $s_{n, j+1}(x)=\left|\frac{\sin (n x-(j+1) \pi)}{n x-(j+1) \pi}\right|=\frac{\sin (n x-j \pi)}{(j+1) \pi-n x}$. Then, by the same reasonings as in the above Case 1$)$, we get

$$
\begin{aligned}
F_{n}(x) & =\frac{\frac{1}{n} \cdot \sin (n x-j \pi)}{\sin (n x-j \pi) /((j+1) \pi-n x)}=\frac{(j+1) \pi-n x}{n} \\
& \leq \frac{(j+1) \pi-(n(j+1 / 2) \pi) / n}{n}=\frac{\pi}{2 n} .
\end{aligned}
$$

Collecting all the above estimates and applying Corollary 3.2 for $\delta_{n}=\frac{\pi}{2 n}$ and from the property $\omega_{1}(f, \lambda \delta)_{[0, \pi]} \leq([\lambda]+1) \omega_{1}(f, \delta)_{[0, \pi]}$, we conclude that the theorem is proved.

Remarks. 1) Note that the order of approximation of the expression $F_{n}(x)$ in Theorem 3.4 cannot be improved. Indeed, for $j \in\{0,1, \ldots, n-1\}$ fixed, take $x=[(j+1 / 2) \pi] / n$. Then, by direct calculation we easily obtain $F_{n}(x)=\frac{\pi}{2 n}$, which implies the desired conclusion.

2) Since

$$
W_{n}^{(M)}(f)(x)=\frac{\bigvee_{k \in I_{n}^{+}(x)} s_{n, k}(x) \cdot f\left(\frac{k \pi}{n}\right)}{\bigvee_{k \in I_{n}^{+}(x)} s_{n, k}(x)}, x \in[0, \pi],
$$

from the proof of Theorem 3.4, Case 1 and Case 2, we immediately can write

$$
\frac{s_{n, k}(x)}{s_{n, j}(x)}=\frac{n x-j \pi}{n} \cdot \frac{1}{\left|x_{n, k}-x\right|}=\frac{x-x_{n, j}}{\left|x_{n, k}-x\right|},
$$

for all $k \in I_{n}^{+}(x), x \in(j \pi / n,(j+1 / 2) \pi / n]$,

and

$$
\frac{s_{n, k}(x)}{s_{n, j+1}(x)}=\frac{(j+1) \pi-n x}{n} \cdot \frac{1}{\left|x_{n, k}-x\right|}=\frac{x_{n, j+1}-x}{\left|x_{n, k}-x\right|},
$$

for all $k \in I_{n}^{+}(x), x \in[(j+1 / 2) \pi / n,(j+1) \pi / n)$, which implies the following piecewise rational form of $W_{n}^{(M)}(f)(x)$, for every $j \in\{0,1, \ldots, n-1\}$,

$$
W_{n}^{(M)}(f)(x)=\left(x-x_{n, j}\right) \bigvee_{k \in I_{n}^{+}(x)} \frac{f\left(\frac{k \pi}{n}\right)}{\left|x_{n, k}-x\right|}, \text { for } x \in(j \pi / n,(j+1 / 2) \pi / n]
$$


and

$$
W_{n}^{(M)}(f)(x)=\left(x_{n, j+1}-x\right) \bigvee_{k \in I_{n}^{+}(x)} \frac{f\left(\frac{k \pi}{n}\right)}{\left|x_{n, k}-x\right|}, \text { for } x \in[(j+1 / 2) \pi / n,(j+1) \pi / n) \text {. }
$$

Here recall that $x_{n, j}=j \pi / n$, for all $j \in\{0,1, \ldots, n\}$.

At the end of this section, we prove that the max-product Whittaker interpolation operator partially preserves the monotonicity on $[0, \pi]$.

Theorem 3.5. (i) If $f:[0, \pi] \rightarrow \mathbb{R}_{+}$is nondecreasing on $[0, \pi]$ then for all $n \in \mathbb{N}$, the max-product Whittaker operator $W_{n}^{(M)}(f)(x)$ is nondecreasing and convex on each subinterval $[j \pi / n,(j+1 / 2) \pi / n]$ and nonincreasing on each subinterval $[(j+1 / 2) \pi / n,(j+1) \pi / n], j \in\{0,1, \ldots, n-1\}$.

(ii) If $f:[0, \pi] \rightarrow \mathbb{R}_{+}$is nonincreasing on $[0, \pi]$ then for all $n \in \mathbb{N}$, the maxproduct Whittaker operator $W_{n}^{(M)}(f)(x)$ is nonincreasing and convex on each subinterval $[(j+1 / 2) \pi / n,(j+1) \pi / n], j \in\{0,1, \ldots, n-1\}$ and nondecreasing on each subinterval $[j \pi / n,(j+1 / 2) \pi / n]$.

Proof. Since $W_{n}^{(M)}(f)$ is continuous on the interval $[0, \pi]$ it will suffice to prove the conclusions of the present theorem on the intervals $(j \pi / n,(j+1 / 2) \pi / n]$ and $[(j+1 / 2) \pi / n,(j+1) \pi / n)$ respectively. Let $W_{n}^{(M)}(f)(x)$ be under the form written by the previous Remark 2 . We can write

$$
\begin{gathered}
W_{n}^{(M)}(f)(x)= \\
\max \left\{\left(x-x_{n, j}\right) \bigvee_{k \in I_{n}^{+}(x), k \leq j} \frac{f\left(\frac{k \pi}{n}\right)}{x-x_{n, k}},\left(x-x_{n, j}\right) \bigvee_{k \in I_{n}^{+}(x), k \geq j+1} \frac{f\left(\frac{k \pi}{n}\right)}{x_{n, k}-x}\right\},
\end{gathered}
$$

for all $x \in(j \pi / n,(j+1 / 2) \pi / n]$, and

$$
\begin{gathered}
W_{n}^{(M)}(f)(x)= \\
\max \left\{\left(x_{n, j+1}-x\right) \bigvee_{k \in I_{n}^{+}(x), k \leq j} \frac{f\left(\frac{k \pi}{n}\right)}{x-x_{n, k}},\left(x_{n, j+1}-x\right) \bigvee_{k \in I_{n}^{+}(x), k \geq j+1}^{n} \frac{f\left(\frac{k \pi}{n}\right)}{x_{n, k}-x}\right\},
\end{gathered}
$$

for all $x \in[(j+1 / 2) \pi / n,(j+1) \pi / n)$.

(i) Suppose that $f:[0, \pi] \rightarrow \mathbb{R}_{+}$is nondecreasing on $[0, \pi]$. Then it is clear that

$$
\left(x-x_{n, j}\right) \bigvee_{k \in I_{n}^{+}(x), k \leq j} \frac{f\left(\frac{k \pi}{n}\right)}{x-x_{n, k}}=f\left(\frac{j \pi}{n}\right), \text { for all } x \in(j \pi / n,(j+1 / 2) \pi / n],
$$

which implies

$$
W_{n}^{(M)}(f)(x)=\max \left\{f\left(\frac{j \pi}{n}\right), \bigvee_{k \in I_{n}^{+}(x), k \geq j+1} \frac{f\left(\frac{k \pi}{n}\right)\left(x-x_{n, j}\right)}{x_{n, k}-x}\right\},
$$


for all $x \in(j \pi / n,(j+1 / 2) \pi / n]$, and

$$
\begin{gathered}
W_{n}^{(M)}(f)(x)= \\
\max \left\{\frac{f\left(\frac{j \pi}{n}\right)\left(x_{n, j+1}-x\right)}{x-x_{n, j}}, \bigvee_{k \in I_{n}^{+}(x), k \geq j+1}^{n} \frac{f\left(\frac{k \pi}{n}\right)\left(x_{n, j+1}-x\right)}{x_{n, k}-x}\right\},
\end{gathered}
$$

for all $x \in[(j+1 / 2) \pi / n,(j+1) \pi / n)$.

Denote $f_{n, k, j}(x)=\frac{x-x_{n, j}}{x_{n, k}-x}, k \geq j+1$. We have

$$
f_{n, k, j}^{\prime}(x)=\frac{x_{n, k}-x_{n, j}}{\left(x-x_{n, k}\right)^{2}}>0 \text { and } f_{n, k, j}^{\prime \prime}(x)=\frac{2\left(x_{n, k}-x_{n, j}\right)}{\left(x_{n, k}-x\right)^{3}}>0,
$$

for all $k \geq j+1$ and $x \in(j \pi / n,(j+1 / 2) \pi / n]$, which implies that $W_{n}^{(M)}(f)(x)$ is nondecreasing and convex on $x \in(j \pi / n,(j+1 / 2) \pi / n]$, as a maximum of nondecreasing and convex functions.

Denote $g_{n, k, j}(x)=\frac{\left(x_{n, j+1}-x\right)}{x_{n, k}-x}, k \geq j+1$. For all $x \in[(j+1 / 2) \pi / n,(j+1) \pi / n)$ and $k \geq j+1$ we have

$$
g_{n, k, j}^{\prime}(x)=\frac{x_{n, j+1}-x_{n, k}}{\left(x_{n, k}-x\right)^{2}} \leq 0 .
$$

Also, denoting $h_{n, j}(x)=\frac{x_{n, j+1}-x}{x-x_{n, j}}$, we get $h^{\prime}(x)=\frac{x_{n, j}-x_{n, j+1}}{\left(x-x_{n, j}\right)^{2}}<0$, for all $x \in$ $[(j+1 / 2) \pi / n,(j+1) \pi / n)$, which implies that $W_{n}^{(M)}(f)(x)$ is nonincreasing on $[(j+1 / 2) \pi / n,(j+1) \pi / n)$, as a maximum of nonincreasing functions.

(ii) Suppose that $f:[0, \pi] \rightarrow \mathbb{R}_{+}$is nonincreasing on $[0, \pi]$. Then it is clear that

$$
\left(x-x_{n, j}\right) \bigvee_{k \in I_{n}^{+}(x), k \geq j+1} \frac{f\left(\frac{k \pi}{n}\right)}{x_{n, k}-x}=\frac{f\left(\frac{(j+1) \pi}{n}\right)\left(x-x_{n, j}\right)}{x_{n, j+1}-x},
$$

for all $x \in(j \pi / n,(j+1 / 2) \pi / n]$, which implies

$$
\begin{gathered}
W_{n}^{(M)}(f)(x)= \\
\max \left\{\left(x-x_{n, j}\right) \bigvee_{k \in I_{n}^{+}(x), k<j} \frac{f\left(\frac{k \pi}{n}\right)}{x-x_{n, k}}, f\left(\frac{j \pi}{n}\right), \frac{f\left(\frac{(j+1) \pi}{n}\right)\left(x-x_{n, j}\right)}{x_{n, j+1}-x}\right\},
\end{gathered}
$$

for all $x \in(j \pi / n,(j+1 / 2) \pi / n]$, and

$$
\begin{aligned}
& W_{n}^{(M)}(f)(x)= \\
& \max \left\{\bigvee_{k \in I_{n}^{+}(x), k \leq j} \frac{x_{n, j+1}-x}{x-x_{n, k}} f\left(\frac{k \pi}{n}\right), f((j+1) \pi / n)\right\},
\end{aligned}
$$


for all $x \in[(j+1 / 2) \pi / n,(j+1) \pi / n)$.

Denote $f_{n, k, j}(x)=\frac{x-x_{n, j}}{x-x_{n, k}}, k<j$. We have

$$
f_{n, k, j}^{\prime}(x)=\frac{x_{n, j}-x_{n, k}}{\left(x-x_{n, k}\right)^{2}}>0
$$

for all $k<j$ and $x \in(j \pi / n,(j+1 / 2) \pi / n]$.

Also, denoting $g_{n, j}(x)=\frac{x-x_{n, j}}{x_{n, j+1}-x}$, for all $x \in(j \pi / n,(j+1 / 2) \pi / n]$ we get

$$
g_{n, j}^{\prime}(x)=\frac{x_{n, j+1}-x_{n, j}}{\left(x_{n, j+1}-x\right)^{2}}>0
$$

which implies that $W_{n}^{(M)}(f)(x)$ is nondecreasing on $(j \pi / n,(j+1 / 2) \pi / n]$ as a maximum of nondecreasing functions.

Now, denoting $g_{n, k, j}(x)=\frac{x_{n, j+1}-x}{x-x_{n, k}}$, for all $k \leq j$ and $x \in[(j+1 / 2) \pi / n,(j+$ 1) $\pi / n$ ) we get

$$
g_{n, k, j}^{\prime}(x)=\frac{x_{n, k}-x_{n, j+1}}{\left(x-x_{n, k}\right)^{2}}<0 \text { and } g_{n, k, j}^{\prime \prime}(x)=-\frac{2\left(x_{n, k}-x_{n, j+1}\right)}{\left(x-x_{n, k}\right)^{3}}>0,
$$

which implies that $W_{n}^{(M)}(f)(x)$ is nonincreasing and convex on $[(j+1 / 2) \pi / n,(j+$ $1) \pi(n)$ as a maximum of nonincreasing and convex functions and proves the theorem.

Remark. In the case of the linear truncated Whittaker operator, there exists an extensive mathematical literature dealing with its convergence, see e.g. Trynin [19] and Sklyarov [16] and the References cited there. In this sense, in [19], Corollary at p. 66, for example it is proved the qualitative result that if $\lim _{n \rightarrow \infty} \omega_{1}(f ; 1 / n) \ln (n)=0$ then $W_{n}(f)(x) \rightarrow f(x)$ uniformly inside of $(0, \pi)$ and pointwise in $[0, \pi]$, while in $[16]$, Theorem 2, p. 190, it is proved the divergence result $\left\|W_{n}(1)-1\right\| \geq \frac{1}{3 \pi}$, for all $n \geq 2$, where $\|\cdot\|$ denotes the uniform norm on $[0, \pi]$.

Comparing all these results with Theorem 3.4, it is clear that the Jacksontype estimate obtained for the max-product Whittaker operator $W_{n}^{(M)}(f)$, is essentially better than the estimates given by its linear correspondent $W_{n}(f)$.

\section{Truncated Max-Product Operator Based on Fejér- Type Kernel}

In this section we deal with the approximation and shape preserving properties of the truncated max-product sampling operator $T_{n}^{(M)}(f)$, given by the formula (3) in Introduction and based on the Fejér-type kernel $\frac{\sin ^{2}(t)}{t^{2}}$.

Remark. Note that since by convention we take $\sin (0) / 0=1$, denoting $s_{n, k}(x)=\frac{\sin ^{2}(n x-k \pi)}{(n x-k \pi)^{2}}$, it is clear that by the obvious property $s_{n, k}(j \pi / n)=1$ 
if $k=j$ and $s_{n, k}(j \pi / n)=0$ if $k \neq j$, we immediately obtain the interpolation properties $T_{n}^{(M)}(f)(j \pi / n)=f(j \pi / n)$, for all $j \in\{0, \ldots, n\}$.

Also, it is clear that $T_{n}^{(M)}(f)(x)$ is a well-defined function for all $x \in[0, \pi]$ and it is continuous on $[0, \pi]$. Indeed, if $x=j \pi / n$ then by $s_{n, j}(j \pi / n)=1$ it follows that $\bigvee_{k=0}^{n} s_{n, k}(j \pi / n) \geq 1>0$, for all $j \in\{0,1, \ldots, n\}$. Now, if $x \in(j \pi / n,(j+$ 1) $\pi / n)$ with $j \in\{0,1, \ldots, n\}$ arbitrary fixed, we easily obtain that $n x-j \pi \in$ $(0, \pi)$ and therefore $s_{n, j}(x)=\frac{\sin ^{2}(n x-j \pi)}{(n x-j \pi)^{2}}>0$. In conclusion, $T_{n}^{(M)}(f)(x)$ is a well-defined function for all $x \in[0, \pi]$ and because both the denominator and numerator are continuous functions as maximum of finite number of continuous functions, it follows the continuity of $T_{n}^{(M)}(f)(x)$ for $x \in[0, \pi]$.

In addition, note that we evidently have $T_{n}\left(e_{0}\right)(x)=e_{0}(x)$, where $e_{0}(x)=1$, for all $x \in[0, \pi]$.

In what follows, we point out that for $f \in C_{+}[0, \pi]$, the $T_{n}^{(M)}$ operator fulfils similar properties with those of the $W_{n}^{(M)}$ operator in Section 3.

Lemma 4.1. (i) We have $T_{n}^{(M)}: C_{+}[0, \pi] \rightarrow C_{+}[0, \pi]$, for all $n \in \mathbb{N}$;

(ii) If $f, g \in C_{+}[0, \pi]$ satisfy $f \leq g$ then $T_{n}^{(M)}(f) \leq T_{n}^{(M)}(g)$ for all $n \in N$;

(iii) $T_{n}^{(M)}(f+g) \leq T_{n}^{(M)}(f)+T_{n}^{(M)}(g)$ for all $f, g \in C_{+}[0, \pi]$;

(iv) For all $f, g \in C_{+}[0, \pi], n \in N$ and $x \in[0, \pi]$ we have

$$
\left|T_{n}^{(M)}(f)(x)-T_{n}^{(M)}(g)(x)\right| \leq T_{n}^{(M)}(|f-g|)(x) ;
$$

(v) $T_{n}^{(M)}$ is positively homogenous, that is $T_{n}^{(M)}(\lambda f)=\lambda T_{n}^{(M)}(f)$ for all $\lambda \geq 0$ and $f \in C_{+}[0, \pi]$.

Proof. (i) The continuity and the positivity of $T_{n}^{(M)}(f)(x)$ on $[0, \pi]$ follows from the previous Remark.

The proofs of (ii)-(v) are exactly the same with the proofs of (ii)-(v) in Lemma 3.1.

Remark. By (3) it is easy to see that instead of (ii), $T_{n}^{(M)}$ satisfies the stronger condition

$$
T_{n}^{(M)}(f \vee g)(x)=T_{n}^{(M)}(f)(x) \vee T_{n}^{(M)}(g)(x), f, g \in C_{+}[0, \pi] .
$$

Corollary 4.2. For all $f \in C_{+}[0, \pi], n \in N$ and $x \in[0, \pi]$ we have

$$
\left|f(x)-T_{n}^{(M)}(f)(x)\right| \leq\left[\frac{1}{\delta} T_{n}^{(M)}\left(\varphi_{x}\right)(x)+1\right] \omega_{1}(f ; \delta)_{[0, \pi]},
$$

where $\delta>0, \varphi_{x}(t)=|t-x|$ for all $t \in[0, \pi], x \in[0, \pi]$ and $\omega_{1}(f ; \delta)_{[0, \pi]}=$ $\max \{|f(x)-f(y)| ; x, y \in[0, \pi],|x-y| \leq \delta\}$.

Proof. Similar to the proof of Corollary 3.2.

We can explicitly write the denominator of $T_{n}^{(M)}(f)(x)$, as follows. 
Lemma 4.3. Denoting $s_{n, k}(x)=\frac{\sin ^{2}(n x-k \pi)}{(n x-k \pi)^{2}}$, for any $j \in\{0,1, \ldots, n-1\}$ we have

$$
\bigvee_{k=0}^{n} s_{n, k}(x)=s_{n, j}(x), \text { for all } x \in\left[\frac{j \pi}{n}, \frac{(j+1 / 2) \pi}{n}\right] \text {, }
$$

and

$$
\bigvee_{k=0}^{n} s_{n, k}(x)=s_{n, j+1}(x), \text { for all } x \in\left[\frac{(j+1 / 2) \pi}{n}, \frac{(j+1) \pi}{n}\right] .
$$

Proof. Let us fix $x \in\left[\frac{j \pi}{n}, \frac{(j+1) \pi}{n}\right]$. From the first Remark of this section we easily obtain $\bigvee_{k=0}^{n} s_{n, k}\left(\frac{j \pi}{n}\right)=s_{n, j}\left(\frac{j \pi}{n}\right)=1$ and $\bigvee_{k=0}^{n} s_{n, k}\left(\frac{(j+1) \pi}{n}\right)=$ $s_{n, j+1}\left(\frac{(j+1) \pi}{n}\right)=1$, therefore, in all what follows we may suppose that $x \in$ $\left(\frac{j \pi}{n}, \frac{(j+1) \pi}{n}\right)$. First, let us notice that $\sin ^{2}(n x-k \pi)=\sin ^{2}(n x-j \pi)$ for all $k \in\{0,1, \ldots, n\}$ which implies $\frac{s_{n, k}(x)}{s_{n, j}(x)}=\frac{(n x-j \pi)^{2}}{(n x-k \pi)^{2}}$ and $\frac{s_{n, k}(x)}{s_{n, j+1}(x)}=\frac{(n x-(j+1) \pi)^{2}}{(n x-k \pi)^{2}}$ for all $k \in\{0,1, \ldots, n\}$. If $k \leq j$ then $0<n x-j \pi \leq n x-k \pi$ which implies $s_{n, k}(x) \leq s_{n, j}(x)$. If $k \geq j+1$ then $0<(j+1) \pi-n x \leq k \pi-n x$ which implies $s_{n, k}(x) \leq s_{n, j+1}(x)$. These results imply $\bigvee_{k=0}^{n} s_{n, k}(x)=\max \left\{s_{n, j}(x), s_{n, j+1}(x)\right\}$. Noting that $\frac{s_{n, j}(x)}{s_{n, j+1}(x)}=\frac{(n x-(j+1) \pi)^{2}}{(n x-j \pi)^{2}}$ and that for $x \in\left[\frac{j \pi}{n}, \frac{(j+1 / 2) \pi}{n}\right]$ we have $0 \leq n x-j \pi \leq(j+1) \pi-n x$ and for $x \in\left[\frac{(j+1 / 2) \pi}{n}, \frac{(j+1) \pi}{n}\right]$ we have $0 \leq$ $(j+1) \pi-n x \leq n x-j \pi$, we immediately obtain the desired conclusion.

In what follows we study the approximation properties of the max-product operator $T_{n}^{(M)}$. For this purpose, it is enough to get a good estimate for the expression

$$
F_{n}(x):=T_{n}^{(M)}\left(\varphi_{x}\right)(x)=\frac{\bigvee_{k=0}^{n} s_{n, k}(x)\left|x_{n, k}-x\right|}{\bigvee_{k=0}^{n} s_{n, k}(x)},
$$

where $s_{n, k}(x)=\frac{\sin ^{2}(n x-k \pi)}{(n x-k \pi)^{2}}$ and $x_{n, k}=k \pi / n$, for all $k \in\{0,1, \ldots, n\}$.

In this sense, we present the following.

Theorem 4.4. If $f:[0, \pi] \rightarrow \mathbb{R}_{+}$is continuous then we have the estimate

$$
\left|T_{n}^{(M)}(f)(x)-f(x)\right| \leq 4 \omega_{1}\left(f ; \frac{1}{n}\right)_{[0, \pi]}, \text { for all } n \in \mathbb{N}, x \in[0, \pi] .
$$

Proof. Let $x \in[0, \pi]$ and let $j \in\{0,1, \ldots, n-1\}$ be such that $x \in$ $\left[\frac{j \pi}{n}, \frac{(j+1) \pi}{n}\right]$. We distinguish the following cases: 1) $x \in\left[\frac{j \pi}{n}, \frac{(j+1 / 2) \pi}{n}\right]$ and 2) $x \in\left[\frac{(j+1 / 2) \pi}{n}, \frac{(j+1) \pi}{n}\right]$. 
Case 1) From Lemma 4.3. we obtain

$$
\begin{aligned}
F_{n}(x) & =\bigvee_{k=0}^{n} \frac{(n x-j \pi)^{2}}{(n x-k \pi)^{2}} \cdot\left|\frac{k \pi}{n}-x\right|=\bigvee_{k=0}^{n} \frac{(n x-j \pi)^{2}}{|n x-k \pi|} \cdot \frac{1}{n} \\
& =\bigvee_{k=0}^{n} \frac{(n x-j \pi)}{|n x-k \pi|} \cdot \frac{(n x-j \pi)}{n} \leq \bigvee_{k=0}^{n} \frac{(n x-j \pi)}{|n x-k \pi|} \cdot \frac{\pi}{2 n}
\end{aligned}
$$

Since $x \in\left[\frac{j \pi}{n}, \frac{(j+1 / 2) \pi}{n}\right]$ it is immediate that $\min \{|n x-k \pi|: k \in\{0,1, \ldots, n\}=$ $n x-j \pi$ (see also the proof of Lemma 4.3.). This implies $\bigvee_{k=0}^{n} \frac{(n x-j \pi)}{|n x-k \pi|} \leq 1$ and we obtain $F_{n}(x) \leq \frac{\pi}{2 n}$.

Case 2) From Lemma 4.3. we obtain

$$
\begin{aligned}
F_{n}(x) & =\bigvee_{k=0}^{n} \frac{(n x-(j+1) \pi)^{2}}{(n x-k \pi)^{2}} \cdot\left|\frac{k \pi}{n}-x\right|=\bigvee_{k=0}^{n} \frac{(n x-(j+1) \pi)^{2}}{|n x-k \pi|} \cdot \frac{1}{n} \\
& =\bigvee_{k=0}^{n} \frac{((j+1) \pi-n x)}{|n x-k \pi|} \cdot \frac{((j+1) \pi-n x)}{n} \leq \bigvee_{k=0}^{n} \frac{((j+1) \pi-n x)}{|n x-k \pi|} \cdot \frac{\pi}{2 n}
\end{aligned}
$$

Since $x \in\left[\frac{(j+1 / 2) \pi}{n}, \frac{(j+1) \pi}{n}\right]$ it is immediate that $\min \{|n x-k \pi|: k \in\{0,1, \ldots, n\}=$ $((j+1) \pi-n x)$ (see also the proof of Lemma 4.3.). This implies $\bigvee_{k=0}^{n} \frac{((j+1) \pi-n x)}{|n x-k \pi|} \leq$ 1 and we obtain $F_{n}(x) \leq \frac{\pi}{2 n}$.

We thus obtain $F_{n}(x) \leq \frac{\pi}{2 n}$ for all $x \in[0,2 \pi]$ and taking $\delta=\frac{\pi}{2 n}$ in Corollary 4.2 ., by the property $\omega_{1}(f, \lambda \delta)_{[0,2 \pi]} \leq([\lambda]+1) \omega_{1}(f, \delta)_{[0,2 \pi]}$ too, we obtain the desired conclusion.

Remarks. 1) Note that the order of approximation of the expression $F_{n}(x)$ in Theorem 4.4 cannot be improved. Indeed, for $j \in\{0,1, \ldots, n-1\}$ fixed, take $x=[(j+1 / 2) \pi] / n$. Then, by direct calculation we easily obtain $F_{n}(x)=\frac{\pi}{2 n}$, which implies the desired conclusion.

At the end of this section, we prove that the max-product interpolation operator $T_{n}^{(M)}(f)$ partially preserves the monotonicity of $f$ on $[0, \pi]$.

Theorem 4.5. (i) If $f:[0, \pi] \rightarrow \mathbb{R}_{+}$is nondecreasing on $[0, \pi]$ then for all $n \in \mathbb{N}$, the truncated max-product Fejér-type operator $T_{n}^{(M)}(f)(x)$ is nondecreasing and convex on each subinterval $[j \pi / n,(j+1 / 2) \pi / n]$ and nonincreasing on each subinterval $[(j+1 / 2) \pi / n,(j+1) \pi / n), j \in\{0,1, \ldots, n-1\}$.

(ii) If $f:[0, \pi] \rightarrow \mathbb{R}_{+}$is nonincreasing on $[0, \pi]$ then for all $n \in \mathbb{N}$, the truncated max-product Fejér-type operator $T_{n}^{(M)}(f)(x)$ is nonincreasing and convex on each subinterval $[(j+1 / 2) \pi / n,(j+1) \pi / n], j \in\{0,1, \ldots, n-1\}$ and nondecreasing on each subinterval $\cdot[j \pi / n,(j+1 / 2) \pi / n]$.

Proof. From the proof of Lemma 4.3. we get

$$
T_{n}^{(M)}(f)(x)=\bigvee_{k=0}^{n} \frac{(n x-j \pi)^{2}}{(n x-k \pi)^{2}} \cdot f\left(\frac{k \pi}{n}\right), x \in\left[\frac{j \pi}{n}, \frac{(j+1 / 2) \pi}{n}\right]
$$


and

$$
T_{n}^{(M)}(f)(x)=\bigvee_{k=0}^{n} \frac{(n x-(j+1) \pi)^{2}}{(n x-k \pi)^{2}} \cdot f\left(\frac{k \pi}{n}\right), x \in\left[\frac{(j+1 / 2) \pi}{n}, \frac{(j+1) \pi}{n}\right]
$$

for all $j \in\{0,1, \ldots, n-1\}$.

(i) Suppose that $f:[0, \pi] \rightarrow \mathbb{R}_{+}$is nondecreasing. If $x \in\left[\frac{j \pi}{n}, \frac{(j+1 / 2) \pi}{n}\right]$ then it is immediate that for $k \leq j$ we have $\frac{(n x-j \pi)^{2}}{(n x-k \pi)^{2}} \cdot f\left(\frac{k \pi}{n}\right) \leq f\left(\frac{k \pi}{n}\right) \leq f\left(\frac{j \pi}{n}\right)$ which implies

$$
T_{n}^{(M)}(f)(x)=\max \left\{f\left(\frac{j \pi}{n}\right), \bigvee_{k=j+1}^{n} \frac{(n x-j \pi)^{2}}{(n x-k \pi)^{2}} \cdot f\left(\frac{k \pi}{n}\right)\right\}
$$

For each $k>j$ let us consider the function $f_{k, n, j}:\left[\frac{j \pi}{n}, \frac{(j+1 / 2) \pi}{n}\right] \rightarrow \mathbb{R}_{+}$, $f_{k, n, j}(x)=\frac{(n x-j \pi)^{2}}{(n x-k \pi)^{2}} \cdot f\left(\frac{k \pi}{n}\right)$. We have $f_{k, n, j}^{\prime}(x)=\frac{-2 n \pi(k-j)(n x-j \pi)}{(n x-k \pi)^{3}} \cdot f\left(\frac{k \pi}{n}\right) \geq 0$ and $f_{k, n, j}^{\prime \prime}(x)=\frac{-2 n^{2} \pi(k-j)(-2 n x-k \pi+3 j \pi)}{(n x-k \pi)^{4}} \cdot f\left(\frac{k \pi}{n}\right) \geq 0$ for all $x \in\left[\frac{j \pi}{n}, \frac{(j+1 / 2) \pi}{n}\right]$. This implies that $T_{n}^{(M)}(f)$ is nondecreasing and convex on $[j \pi / n,(j+1 / 2) \pi / n]$, as a maximum of nondecreasing and convex functions.

If $x \in\left[\frac{(j+1 / 2) \pi}{n}, \frac{(j+1) \pi}{n}\right]$ then it is immediate that $0 \leq(j+1) \pi-n x \leq n x-$ $j \pi \leq n x-k \pi$ for all $k \leq j$. This implies $\frac{(n x-(j+1) \pi)^{2}}{(n x-k \pi)^{2}} \cdot f\left(\frac{k \pi}{n}\right) \leq \frac{(n x-j \pi)^{2}}{(n x-k \pi)^{2}} \cdot f\left(\frac{k \pi}{n}\right) \leq$ $f\left(\frac{k \pi}{n}\right) \leq f\left(\frac{j \pi}{n}\right) \leq f\left(\frac{(j+1) \pi}{n}\right)$ for all $k \leq j$. Therefore, we obtain

$$
T_{n}^{(M)}(f)(x)=\max \left\{f\left(\frac{(j+1) \pi}{n}\right), \bigvee_{k=j+2}^{n} \frac{(n x-(j+1) \pi)^{2}}{(n x-k \pi)^{2}} \cdot f\left(\frac{k \pi}{n}\right)\right\}
$$

For each each $k>j+1$ let us consider the functions $g_{k, n, j}:\left[\frac{(j+1 / 2) \pi}{n}, \frac{(j+1) \pi}{n}\right] \rightarrow$ $\mathbb{R}_{+}, g_{k, n, j}(x)=\frac{(n x-(j+1) \pi)^{2}}{(n x-k \pi)^{2}} \cdot f\left(\frac{k \pi}{n}\right)$. We have $g_{k, n, j}^{\prime}(x)=\frac{-2 n \pi(k-j-1)(n x-(j+1) \pi)}{(n x-k \pi)^{3}}$. $f\left(\frac{k \pi}{n}\right) \leq 0$ for all $x \in\left[\frac{(j+1 / 2) \pi}{n}, \frac{(j+1) \pi}{n}\right]$ which implies implies that $T_{n}^{(M)}(f)$ is nonincreasing on $[(j+1 / 2) \pi / n,(j+1) \pi / n]$ as a maximum of nonincreasing functions.

(ii) Suppose that $f:[0, \pi] \rightarrow \mathbb{R}_{+}$is nonincreasing. If $x \in\left[\frac{j \pi}{n}, \frac{(j+1 / 2) \pi}{n}\right]$ then it is immediate that for $k \geq j+1$ we have $0 \leq n x-j \pi \leq(j+1) \pi-n x \leq k \pi-n x$ which implies $\frac{(n x-j \pi)^{2}}{(n x-k \pi)^{2}} \cdot f\left(\frac{k \pi}{n}\right) \leq f\left(\frac{k \pi}{n}\right) \leq f\left(\frac{j \pi}{n}\right)$ and consequently we get

$$
T_{n}^{(M)}(f)(x)=\max \left\{f\left(\frac{j \pi}{n}\right), \bigvee_{k=0}^{j-1} \frac{(n x-j \pi)^{2}}{(n x-k \pi)^{2}} \cdot f\left(\frac{k \pi}{n}\right)\right\}
$$


For each $k<j$ let us consider the function $f_{k, n, j}:\left[\frac{j \pi}{n}, \frac{(j+1 / 2) \pi}{n}\right] \rightarrow \mathbb{R}_{+}$, $f_{k, n, j}(x)=\frac{(n x-j \pi)^{2}}{(n x-k \pi)^{2}} \cdot f\left(\frac{k \pi}{n}\right)$. It is immediate that $f_{k, n, j}^{\prime}(x) \leq 0$ and $f_{k, n, j}^{\prime \prime}(x) \geq 0$ for all $x \in\left[\frac{j \pi}{n}, \frac{(j+1 / 2) \pi}{n}\right]$ (see the proof of case (i)). Therefore, $T_{n}^{(M)}(f)$ is nonincreasing and convex on $\left[\frac{j \pi}{n}, \frac{(j+1 / 2) \pi}{n}\right]$ as a maximum of nonincreasing and convex functions.

If $x \in\left[\frac{(j+1 / 2) \pi}{n}, \frac{(j+1) \pi}{n}\right]$ then, for $k \geq j+1$ we have $0 \leq(j+1) \pi-n x \leq k \pi-n x$ which implies $\frac{(n x-(j+1) \pi)^{2}}{(n x-k \pi)^{2}} \cdot f\left(\frac{k \pi}{n}\right) \leq f\left(\frac{k \pi}{n}\right) \leq f\left(\frac{j \pi}{n}\right)$. This implies

$$
T_{n}^{(M)}(f)(x)=\max \left\{f\left(\frac{j \pi}{n}\right), \bigvee_{k=0}^{j-1} \frac{((j+1) \pi-n x)^{2}}{(n x-k \pi)^{2}} \cdot f\left(\frac{k \pi}{n}\right)\right\} .
$$

For each $k<j$ let us consider the functions $g_{k, n, j}:\left[\frac{(j+1 / 2) \pi}{n}, \frac{(j+1) \pi}{n}\right] \rightarrow \mathbb{R}_{+}$, $g_{k, n, j}(x)=\frac{(n x-(j+1) \pi)^{2}}{(n x-k \pi)^{2}} \cdot f\left(\frac{k \pi}{n}\right)$. It is immediate that $g_{k, n, j}^{\prime}(x) \geq 0$ for all $x \in$ $\left[\frac{(j+1 / 2) \pi}{n}, \frac{(j+1) \pi}{n}\right]$ (see the proof of case (i)) and this implies that $T_{n}^{(M)}(f)$ is nondecreasing on $\left[\frac{(j+1 / 2) \pi}{n}, \frac{(j+1) \pi}{n}\right]$ as a maximum of nondecreasing functions.

\section{ACKNOWLEDGEMENTS}

The authors thank the referees for reading so carefully the manuscript and for their useful remarks.

The work of the first author was supported by the Sectoral Operational Programme for Human Resources Development 2007-2013, co-financed by the European Social Fund, under the project number POSDRU/-/107/1.5/S/76841 with the title "Modern Doctoral Studies:Internationalization and Interdisciplinarity".

The work of both authors was supported by a grant of the Romanian National Authority for Scientific Research, CNCS-UEFISCDI, project number PN-II-IDPCE-2011-3-0861.

\section{References}

[1] C. Bardaro, P.L. Butzer, R.L. Stens and G. Vinti, Approximation error of the Whittaker cardinal series in terms of an averaged modulus of smoothness covering discontinuous signals, J. Math. Anal. Appl., 316, 269-306, 2006.

[2] C. Bardaro, P.L. Butzer, R.L. Stens and G. Vinti, Kantorovich-type generalized sampling series in the setting of Orlicz spaces, Sampl. Theory Signal Image Process., 6(1), 19-52, 2007. 
[3] C. Bardaro, P. L. Butzer, R. L. Stens and G. Vinti, Prediction by samples from the past with error estimates covering discontinuous signals, IEEE Trans. on Information Theory, 56(1), 614-633, 2010.

[4] B. Bede and S. G. Gal, Approximation by nonlinear Bernstein and FavardSzász-Mirakjan operators of max-product kind, Journal of Concrete and Applicable Mathematics, 8(2), 193-207, 2010.

[5] B. Bede, L. Coroianu and S. G. Gal, Approximation and shape preserving properties of the Bernstein operator of max-product kind, Intern. J. Math. Math. Sci., vol. 2009, Article ID 590589, 26 pages, 2009. doi: $10.1155 / 2009 / 590589$.

[6] E. Borel, Sur l'interpolation, C.R. Acad. Sci. Paris, 124, 673-676, 1897.

[7] P. L. Butzer, A survey of the Whittaker-Shannon sampling theorem and some of its extensions, J. Math. Res. Expos., 3, 185-212, 1983.

[8] P. L. Butzer, W. Engels, S. Ries and R. L. Stens, The Shannon sampling series and the reconstruction of signals in terms of linear, quadratic and cubic splines, SIAM Journal on Applied Mathematics, 46(2), 299-323, 1986.

[9] P. L. Butzer, W. Splettstö $\beta$ er and R. L. Stens, The sampling theorems and linear prediction in signal analysis, Jahresber. Deutsch. Math-Verein, 90, 1-70, 1988.

[10] P. L. Butzer and R. L. Stens, The Poisson summation formula, Whittaker's cardinal series and approximate integration, in : Proc. Second Edmonton Conference on Approximation Theory, Canadian Mathematical Society, 3, 19-36, 1983.

[11] L. Coroianu and S. G. Gal, Approximation by nonlinear generalized sampling operators of max-product kind, Sampl. Theory Signal Image Process., 9(1-3), 59-75, 2010.

[12] S. G. Gal, Shape-Preserving Approximation by Real and Complex Polynomials, Birkhäuser, Boston-Basel-Berlin, 2008.

[13] A. Kivinukk and G. Tamberg, Interpolating generalized Shannon sampling operators, their norms and approximation properties, Sampl. Theory Signal Image Process., 8(1), 77-95, 2009.

[14] A. Kivinukk and G. Tamberg, On approximation properties of sampling operators by dilated kernels, 8th Intern. Conf. on Sampling Theory and Applications, SampTA'09, Marseille, May 18-22, 2009, Poster sessions, electronic access at www.latp.univ-mrs.fr/SAMPTA09/FinalSubmissions/187.pdf 
[15] G. Plana, Sur une nouvelle expression analytique des nombers Bernoulliens, Academia di Torino, 25, 403-418, 1820.

[16] V. P. Sklyarov, On the best uniform sinc-approximation on a finite interval, East J. Approx., 14(2), 183-192, 2008.

[17] R. L. Stens, Approximation of functions by Whittaker's cardinal series, in : General Inequalities 4, Proc. Conference, Oberwolfach, Germany, May 1983, W. Walter ed., ISNM 71, Birkhauser Verlag, Basel, pp. 137-149, 1984

[18] M. Theis, Über eine Interpolationsformel von de la Vallée-Poussin, Math. Z., 3, 93-113, 1919.

[19] A. Yu. Trynin, A criterion for the uniform convergence of sincapproximation on a segment, Russian Mathematics (Iz. VUZ), 52(6), 58-69, 2008.

[20] E. T. Whittaker, On the functions which are represented by expansions of the interpolation theory, Proc. Roy. Soc. Edinburgh, 35, 181-194, 1915. 